\title{
THE INDENTATION OF AN ANISOTROPIC HALF SPACE BY A RIGID PUNCH
}

\author{
D. L. CLEMENTS
}

(Received 6 June 1969; revised 6 October 1969)

Communicated by A. F. Pillow

Eshelby et al. [1] and Stroh [2] have developed the theory of anisotropic elasticity for a three dimensional state of stress in which the stress is independent of one of the Cartesian coordinates. Various problems involving dislocations in an infinite anisotropic medium are solved in the first paper, while Stroh considers dislocation problems as well as determining the stresses round a crack subjected to an arbitrary non-uniform applied stress. In this note, which follows their treatment, we consider the problem of determining the stresses produced by the indentation of the plane surface of an anisotropic half space by a rigid punch. Problems of this type have been solved by Green and Zerna [3], Lekhnitskii [4], Brilla [5], Gallin [6] and Milne-Thomson [7] but if we take Cartesian coordinates $x_{1}, x_{2}, x_{3}$ and let the stress be independent of $x_{3}$, then these authors all assume the $x_{1} x_{2}$ plane to be one of elastic symmetry. The solution presented in this paper does not require this assumption so that it has a more general application than has been the case with previous solutions to problems of this type. The first part of the analysis given here is for general anisotropy, but in order to obtain a solution to the punch problem by the method of this paper, it is necessary to consider only a particular class of anisotropic materials. The indentation of such materials by a circular block is discussed in section 4 and the results are used in section 5 to examine the case when the circular block is on a transversely isotropic half space.

\section{General equations}

The stresses $\sigma_{i j}$ are related to the elastic displacements $u_{k}$ by the equations

$$
\sigma_{i j}=c_{i j k l} \frac{\partial u_{k}}{\partial x_{l}}
$$

where $i, j, k, l=1,2,3$ and the convention of summing over a repeated Latin suffix is used. The elastic moduli $c_{i j k l}$ have the symmetry properties

$$
c_{i j k l}=c_{j i k l}=c_{i j l k}=c_{k l i j}
$$


On substituting (1) in the equilibrium equations

we obtain

$$
\frac{\partial \sigma_{i j}}{\partial x_{j}}=0
$$

$$
c_{i j k l} \frac{\partial^{2} u_{k}}{\partial x_{j} \partial x_{l}}=0
$$

Now we suppose that $u_{k}$ is independent of $x_{3}$, and, following Eshelby et al. take

$$
u_{k}=A_{k} f\left(x_{1}+p x_{2}\right)
$$

where $f(z)$ is an analytic function of the complex variable $z ;(5)$ is a solution of the equations (4) provided the constant vector $A_{k}$ satisfies the equations

$$
\left(c_{i 1 k 1}+p c_{i 1 k 2}+p c_{i 2 k 1}+p^{2} c_{i 2 k 2}\right) A_{k}=0
$$

Values of $A_{k}$, not identically zero, can be found to satisfy these equations if $p$ is a root of the sextic equation

$$
\left|c_{i 1 k 1}+p c_{i 1 k 2}+p c_{i 2 k 1}+p^{2} c_{i 2 k 2}\right|=0
$$

By applying the condition that the strain energy density

$$
c_{i j k l} \frac{\partial u_{i}}{\partial x_{j}} \frac{\partial u_{k}}{\partial x_{l}}
$$

should be positive for any state of strain Eshelby $e t$ al. have proved that equation (7) has no real root, so that the roots occur in complex conjugate pairs. The three roots with positive imaginary part will be denoted by $p_{\alpha}(\alpha=1,2,3)$ with complex conjugates $\bar{p}_{\alpha}$; the corresponding values of $A_{k}$ obtained from equation (6) are $A_{k \alpha}$ and $\bar{A}_{k x}$. Summation over $\alpha$ will always be indicated explicitly. It will be assumed that the roots $p_{\alpha}$ are all distinct; equal roots may be regarded as the limiting case of distinct roots. A general expression for the displacement may then be written

$$
u_{k}=\sum_{\alpha} A_{k \alpha} f_{\alpha}\left(z_{\alpha}\right)+\sum_{\alpha} \overline{A_{k \alpha}} \overline{f_{\alpha}}\left(\overline{z_{\alpha}}\right)
$$

where $z_{\alpha}=x_{1}+p_{\alpha} x_{2}$. From (1) we write the stresses as

$$
\sigma_{i j}=\sum_{\alpha} L_{i j \alpha} f_{\alpha}^{\prime}\left(z_{\alpha}\right)+\sum_{\alpha} \overline{L_{i j \alpha}} \overline{f_{\alpha}^{\prime}}\left(\overline{z_{\alpha}}\right)
$$

where

$$
L_{i j \alpha}=\left(c_{i j k 1}+p_{\alpha} c_{i j k 2}\right) A_{k \alpha}
$$

and dashes denote differentiation with respect to $z_{\alpha}$. 


\section{Indentation by a rigid punch}

Consider an anisotropic elastic body which occupies the half space $x_{2}<0$. Let the area of contact between the punch and the elastic material be

$$
-b<x_{1}<a, \quad-\infty<x_{3}<\infty
$$

where $a$ and $b$ are positive constants. Then the boundary conditions on the plane $x_{2}=0$ are

$$
\begin{array}{ll}
\sigma_{12}\left(x_{1}, 0\right)=\sigma_{23}\left(x_{1}, 0\right)=0 & -\infty<x_{1}<\infty \\
\sigma_{22}\left(x_{1}, 0\right)=0 & x_{1}<-b, \quad x_{1}>a
\end{array}
$$

and on $-b<x_{1}<a$ only the normal component of displacement is given, so that

$$
u_{2}\left(x_{1}, 0\right)=g\left(x_{1}\right) \quad-b<x_{1}<a
$$

with

$$
\int_{-b}^{a} \sigma_{22}\left(x_{1}, 0\right) d x_{1}=-P
$$

where $P$ is the total applied force per unit length.

Let $f_{\alpha}\left(z_{\alpha}\right)=F_{\alpha} \phi\left(z_{\alpha}\right)$ where $F_{\alpha}$ is a complex constant and $\phi(z)$ is analytic in $x_{2}<0$. Hence using (9) and (10) the components of stress and displacement are

$$
\begin{aligned}
u_{k} & =\sum_{\alpha} A_{k \alpha} F_{\alpha} \phi\left(z_{\alpha}\right)+\sum_{\alpha} \overline{A_{k \alpha}} \overline{F_{\alpha}} \bar{\phi}\left(\overline{z_{\alpha}}\right) \\
\sigma_{i j} & =\sum_{\alpha} L_{i j \alpha} F_{\alpha} \phi^{\prime}\left(z_{\alpha}\right)+\sum_{\alpha} \overline{L_{i j \alpha}} \overline{F_{\alpha}} \phi^{\prime}\left(\overline{z_{\alpha}}\right)
\end{aligned}
$$

The boundary conditions (13) will be satisfied if

$$
\sum_{\alpha} L_{12 \alpha} F_{\alpha}=0, \quad \sum_{\alpha} L_{23 \alpha} F_{\alpha}=0
$$

Suppose that there exist values of $F_{\alpha}$ such that the equations (19) are satisfied and

$$
\sum_{\alpha} A_{2 \alpha} F_{\alpha}=i M, \quad \sum_{\alpha} L_{22 \alpha} F_{\alpha}=N
$$

where $M$ and $N$ are real constants. Then using (17) the condition (15) may be written as

$$
\operatorname{Re}\left[i M \phi\left(x_{1}\right)\right]=\frac{1}{2} g\left(x_{1}\right) \quad-b<x_{1}<a
$$

Also using (18) and (20) it follows that on the boundary $x_{2}=0$

$$
\sigma_{22}\left(x_{1}, 0\right)=2 N \operatorname{Re}\left[\phi^{\prime}\left(x_{1}\right)\right]
$$

so that conditions (14) and (16) may be used to give

$$
\begin{aligned}
& \operatorname{Re}\left[\phi\left(x_{1}\right)\right]=0 \quad x_{2}=0, \quad x_{1}<-b \\
& =-\frac{1}{2} P / N \quad x_{2}=0, \quad x_{1}>a
\end{aligned}
$$


The information (21) and (22) may be written in a single equation

$$
\begin{array}{rlrl}
\operatorname{Re}\left[\frac{\phi\left(x_{1}\right)}{\left(x_{1}-a\right)^{\frac{1}{2}}\left(x_{1}+b\right)^{\frac{1}{2}}}\right] & =0 \quad x_{1}<-b & \\
& =\frac{\frac{1}{2} g\left(x_{1}\right)}{M\left(a-x_{1}\right)^{\frac{1}{2}}\left(x_{1}+b\right)^{\frac{1}{2}}} & & -b<x_{1}<a \\
& =\frac{-P}{2 N\left(x_{1}-a\right)^{\frac{1}{2}}\left(x_{1}+b\right)^{\frac{1}{2}}} & x_{1}>a
\end{array}
$$

Use of Cauchy's theorem in the form

$$
f(z)=-\frac{1}{\pi i} \int_{-\infty}^{\infty} \frac{\operatorname{Re} f\left(x_{1}\right)}{x_{1}-z} d x_{1}
$$

for a function analytic in the lower half plane, gives

$$
\begin{aligned}
\frac{\phi\left(z_{\alpha}\right)}{\left(z_{\alpha}-a\right)^{\frac{1}{2}}\left(z_{\alpha}+b\right)^{\frac{1}{2}}}= & \frac{1}{\pi i} \int_{-b}^{a} \frac{-\frac{1}{2} g\left(x_{1}\right)}{M\left(a-x_{1}\right)^{\frac{1}{2}}\left(x_{1}+b\right)^{\frac{1}{2}}\left(x_{1}-z_{\alpha}\right)} d x_{1} \\
& +\frac{1}{\pi i} \int_{a}^{\infty} \frac{P}{2 N\left(x_{1}-a\right)^{\frac{1}{2}}\left(x_{1}+b\right)^{\frac{1}{2}}\left(x_{1}-z_{\alpha}\right)} d x_{1}
\end{aligned}
$$

where on the left, $0 \geqq \arg \left(z_{\alpha}-a\right)^{\frac{1}{2}} \geqq-\frac{1}{2} \pi, 0 \geqq \arg \left(z_{\alpha}+b\right)^{\frac{1}{2}} \geqq-\frac{1}{2} \pi$ for $\operatorname{Im} z_{\alpha}$ $<0$. Hence

$$
\begin{aligned}
\phi\left(z_{\alpha}\right)= & \frac{\left(z_{\alpha}-a\right)^{\frac{1}{2}}\left(z_{\alpha}+b\right)^{\frac{1}{2}}}{\pi i M} \int_{-b}^{a} \frac{-\frac{1}{2} g\left(x_{1}\right)}{\left(a-x_{1}\right)^{\frac{1}{2}}\left(x_{1}+b\right)^{\frac{1}{2}}\left(x_{1}-z_{\alpha}\right)} d x_{1} \\
& -\frac{P}{\pi i N} \log \left\{i(a+b)^{-\frac{1}{2}}\left[\left(z_{\alpha}-a\right)^{\frac{1}{2}}+\left(z_{\alpha}+b\right)^{\frac{1}{2}}\right]\right\} .
\end{aligned}
$$

\section{Restriction to a particular class of anisotropic materials}

The solution presented in section 2 is only applicable if suitable values of the $F_{\alpha}$ can be found to satisfy equations (19) and (20). We must therefore restrict our attention to the class of anisotropic materials for which such values of the $F_{\alpha}$ can be found. In this section we show that it is possible to find suitable $F_{\alpha}$ for the class of materials for which the constants $c_{1112}, c_{1222}, c_{1123}, c_{1213}, c_{2223}$ and $c_{1323}$ are zero. This is not necessarily the most general class of materials for which the solution of section 2 is valid, but with these six constants zero the sextic in $p(7)$ becomes a cubic in $p^{2}$ and with this simplification it is reasonably easy to establish the existence of values of the $F_{\alpha}$ to satisfy (19) and (20). Also it is a class of materials of some interest since it includes orthotropy and transverse isotropy as special cases. 
Putting the six constants equal to zero the matrix equation (6) reduces to

$$
\left[\begin{array}{lll}
c_{1111}+p^{2} c_{1212} & \left(c_{1122}+c_{1221}\right) p & c_{1131}+p^{2} c_{1232} \\
\left(c_{1122}+c_{1221}\right) p & c_{1212}+p^{2} c_{2222} & \left(c_{2231}+c_{2132}\right) p \\
c_{1131}+p^{2} c_{3212} & \left(c_{3122}+c_{3211}\right) p & c_{3131}+p^{2} c_{3232}
\end{array}\right]\left[\begin{array}{l}
A_{1} \\
A_{2} \\
A_{3}
\end{array}\right]=0
$$

where the values of $p$ are obtained by solving equation (7) which is a cubic in $p^{2}$. We consider two possible cases.

CASE 1. Suppose the roots of the cubic are real. Then since $p$ cannot be real the cubic in $p^{2}$ must have negative roots. Hence the six values of $p$ are complex numbers with zero real part. Then if the $A_{3 \alpha}$ are put equal to 1 equation (25) has a solution for which the $A_{1 \alpha}$ have zero imaginary part and the $A_{2 \alpha}$ have zero real part. Also using (11) it may easily be shown that with this choice of the $A_{i x}$ the $L_{12 \alpha}$ and $L_{23 \alpha}$ have zero real part and the $L_{22 \alpha}$ have zero imaginary part. Hence we may choose $F_{3}=1$ and then real and unique values of $F_{1}$ and $F_{2}$ may be obtained from (19) provided $L_{121} L_{232}-L_{122} L_{231} \neq 0$. Values of $M$ and $N$ may then be obtained from (20).

CASE 2. Suppose only one of the roots ( $p_{1}$ say) of the cubic is real and negative. Then the other roots form a conjugate pair and $p_{2}=-\bar{p}_{3}$. Putting the $A_{3 \alpha}$ equal to 1 equation (25) has a solution for which $A_{11}$ has zero imaginary part and $A_{21}$ has zero real part. Also $A_{22}=-\bar{A}_{23}$ and $A_{12}=\bar{A}_{13}$. Then using (11) it may easily be shown that $L_{121}$ and $L_{231}$ have zero real part and that $L_{122}=-\bar{L}_{123}$ and $L_{232}=-\bar{L}_{233}$. Also $L_{221}$ has zero imaginary part and $L_{222}=L_{223}$. Hence we may choose $F_{1}=1$ and then unique values of $F_{2}$ and $F_{3}$ such that $F_{2}=\bar{F}_{3}$ may be obtained from (19) provided $L_{122} L_{233}-L_{123} L_{232} \neq 0$. Values of $M$ and $N$ may then be obtained from (20).

\section{Indentation by a circular block}

Here we take

$$
g\left(x_{1}\right)=\mathscr{B} x_{1}^{2}-\gamma
$$

which corresponds to the case of a rigid circular punch of large radius $R$ with $\mathscr{B}=1 / 2 R$. Substituting for $g\left(x_{1}\right)$ in (24) and integrating we get

$$
\begin{aligned}
\phi\left(z_{\alpha}\right)= & +\frac{i}{2 M}\left[\frac{1}{2} \mathscr{B}\left(a-b+2 z_{\alpha}\right)\left(z_{\alpha}-a\right)^{\frac{1}{2}}\left(z_{\alpha}+b\right)^{\frac{1}{2}}-\mathscr{B} z_{\alpha}^{2}+\gamma\right] \\
& -\frac{P}{\pi i N} \log \left\{i(a+b)^{-\frac{1}{2}}\left[\left(z_{\alpha}-a\right)^{\frac{1}{2}}+\left(z_{\alpha}+b\right)^{\frac{1}{2}}\right]\right\}
\end{aligned}
$$

and it is easily checked that this function has the required property (23) and is $O\left(\log \left|z_{\alpha}\right|\right)$ as $\left|z_{\alpha}\right| \rightarrow \infty$. For this particular case we require that the stresses are 
finite at the ends $a, b$ of the arc of contact of the punch with the elastic half space. This will be the case if $\phi^{\prime}\left(z_{\alpha}\right)$ is finite at $z_{\alpha}=a,-b$. Differentiate (27) with respect to $z_{\alpha}$. Then the coefficient of $\left(z_{\alpha}-a\right)^{-\frac{1}{2}}\left(z_{\alpha}+b\right)^{-\frac{1}{2}}$ is

$$
+\frac{1}{4} i \frac{\mathscr{B}}{2 M}\left(4 z_{\alpha}^{2}-(a-b)^{2}\right)-\frac{P}{2 \pi i N}
$$

For finite stresses, this expression must vanish at $z_{\alpha}=a, z_{\alpha}=-b$ and this condition will be satisfied if

$$
a=b=\left\{\frac{-P M}{\pi N \mathscr{B}}\right\}^{\frac{1}{2}} .
$$

\section{A circular block on a transversely isotropic solid}

It follows from equation (28) that for a circular punch the arc of contact with the half space is proportional to $(-M / N)^{\frac{1}{2}}$. In this section we calculate $(-M / N)^{\frac{1}{2}}$ for a circular punch on a transversely isotropic half space with the boundary of the half space normal to the transverse plane. The elastic behaviour of transversely isotropic materials is characterized by five elastic constants which will be denoted by $A, N, F, C$ and $L$. If the $x_{2}$-axis lies in the transverse plane and the $x_{1}$-axis makes an angle $\alpha$ with the transverse plane (Figure 1 ) then the only non zero $c_{i j k l}$ which are of interest are given by

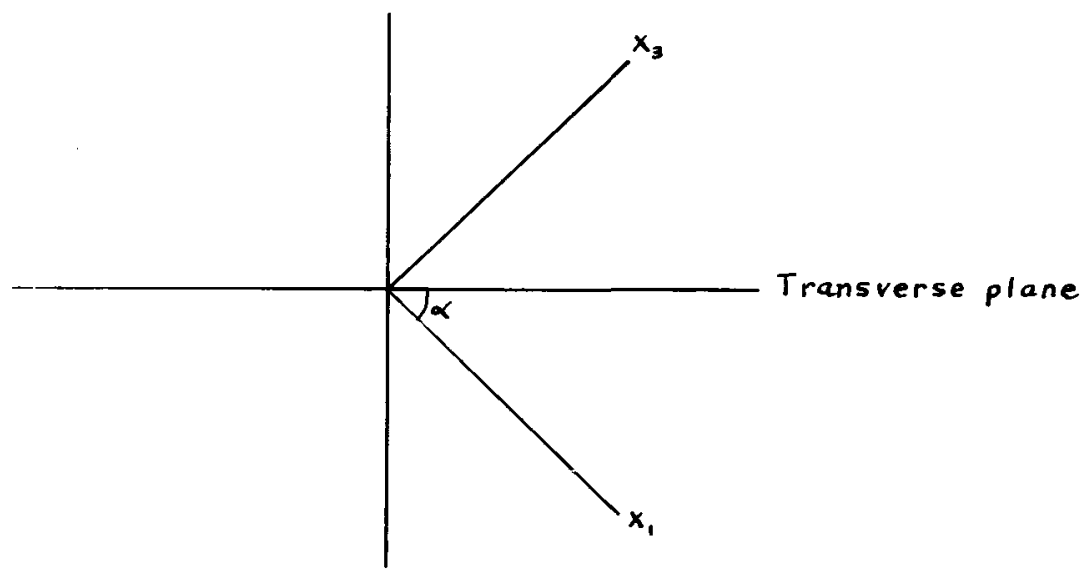

Figure 1

$$
\begin{aligned}
& c_{1111}=A \cos ^{4} \alpha+2(F+2 L) \cos ^{2} \alpha \sin ^{2} \alpha+C \sin ^{4} \alpha \\
& c_{1122}=N \cos ^{2} \alpha+F \sin ^{2} \alpha \\
& c_{1131}=(A-F-2 L) \cos ^{3} \alpha \sin \alpha+(F-C+2 L) \cos \alpha \sin ^{3} \alpha \\
& c_{2222}=A, \quad c_{2231}=(N-F) \cos \alpha \sin \alpha
\end{aligned}
$$




$$
\begin{aligned}
& c_{3311}=(A+C-4 L) \cos ^{2} \alpha \sin ^{2} \alpha+F\left(\cos ^{4} \alpha+\sin ^{4} \alpha\right) \\
& c_{3322}=F \cos ^{2} \alpha+N \sin ^{2} \alpha \\
& c_{3331}=(A-F-2 L) \cos \alpha \sin ^{3} \alpha+(F-C+2 L) \cos ^{3} \alpha \sin \alpha \\
& c_{1331}=L\left(\cos ^{2} \alpha-\sin ^{2} \alpha\right)^{2}-(2 F-C-A) \cos ^{2} \alpha \sin ^{2} \alpha \\
& c_{2332}=L \cos ^{2} \alpha+\frac{1}{2}(A-N) \sin ^{2} \alpha \\
& c_{2312}=\left(\frac{1}{2}(A-N)-L\right) \cos \alpha \sin \alpha \\
& c_{1212}=\frac{1}{2}(A-N) \cos ^{2} \alpha+L \sin ^{2} \alpha
\end{aligned}
$$

Consider firstly the special cases when $\alpha=0$ and $\frac{1}{2} \pi$. For transversely isotropic materials equation (7) is a cubic in $p^{2}$ and when $\alpha=0$ the cubic has three equal roots so the solution to the punch problem may be obtained by comparison with the solution for the isotropic case. When $\alpha=\frac{1}{2} \pi$ (25) becomes

$$
\left[\begin{array}{ccc}
C+L p^{2} & (F+L) p & 0 \\
(F+L) p & L+A p^{2} & 0 \\
0 & 0 & L+\frac{1}{2}(A-N) p^{2}
\end{array}\right]\left[\begin{array}{l}
A_{1} \\
A_{2} \\
A_{3}
\end{array}\right]=0
$$

Also the determinant (7) may be expanded to give

$$
\left(\frac{1}{2}(A-N) p^{2}+L\right)\left(A L p^{4}-\left(F^{2}+2 F L-A C\right) p^{2}+C L\right)=0
$$

Let $p_{1}^{2}=-2 L /(A-N)$. Then $A_{11}=A_{21}=0$ and $A_{31}$ is arbitrary so let $A_{31}=0$. If the roots of the quadratic term are denoted by $p_{2}^{2}$ and $p_{3}^{2}$ then choosing $A_{22}=$ $A_{23}=i$ it follows that

$$
\begin{gathered}
A_{12}=\frac{-i(F+L) p_{2}}{C+L p_{2}^{2}}, \quad A_{13}=\frac{-i(F+L) p_{3}}{C+L p_{3}^{2}} \\
A_{32}=A_{33}=0 .
\end{gathered}
$$

Also using equation (11) $L_{23 \alpha}=0$ and

$$
\begin{aligned}
& L_{121}=0, \quad L_{122}=i L\left[\frac{C-F p_{2}^{2}}{C+L p_{2}^{2}}\right], \quad L_{123}=i L\left[\frac{C-F p_{3}^{2}}{C+L p_{3}^{2}}\right] \\
& L_{221}=0, \quad L_{222}=i p_{2}\left[A-\frac{F(F+L)}{C+L p_{2}^{2}}\right], \quad L_{223}=i p_{3}\left[A-\frac{F(F+L)}{C+L p_{3}^{2}}\right]
\end{aligned}
$$

If $p_{2}^{2}$ and $p_{3}^{2}$ are real then real values of $F_{2}$ and $F_{3}$ which satisfy (19) are given by

$$
F_{2}=\frac{C+L p_{2}^{2}}{C-F p_{2}^{2}}, \quad F_{3}=\frac{-\left(C+L p_{3}^{2}\right)}{C-F p_{3}^{2}}
$$


Hence

$$
\begin{aligned}
& M=\frac{C+L p_{2}^{2}}{C-F p_{2}^{2}}-\frac{C+L p_{3}^{2}}{C-F p_{3}^{2}} \\
& N=i p_{2}\left[\frac{A\left(C+L p_{2}^{2}\right)-F(F+L)}{C-F p_{2}^{2}}\right]-i p_{3}\left[\frac{A\left(C+L p_{3}^{2}\right)-F(F+L)}{C-F p_{3}^{2}}\right]
\end{aligned}
$$

If $p_{2}^{2}$ and $p_{3}^{2}$ are complex conjugates and $p_{2}=-\bar{p}_{3}$ then suitable values of $F_{2}$ and $F_{3}$ which satisfy (19) are given by

Hence

$$
F_{2}=i\left[\frac{C+L p_{2}^{2}}{C-F p_{2}^{2}}\right], \quad F_{3}=-i\left[\frac{C+L p_{3}^{2}}{C-F p_{3}^{2}}\right]
$$

$$
\begin{gathered}
i M=-\frac{C+L p_{2}^{2}}{C-F p_{2}^{2}}+\frac{C+L p_{3}^{2}}{C-F p_{3}^{2}} \\
N=-p_{2}\left[\frac{A\left(C+L p_{2}^{2}\right)-F(F+L)}{C-F p_{2}^{2}}\right]+p_{3}\left[\frac{A\left(C+L p_{3}^{2}\right)-F(F+L)}{C-F p_{3}^{2}}\right]
\end{gathered}
$$

When $0<\alpha<\frac{1}{2} \pi$ the calculation of $M$ and $N$ becomes more complicated. However the task may be carried out reasonably easily with the aid of a computer and this has been done for zinc which has the elastic constants $A=16.5, N=3.1$, $F=5, C=6.2$ and $L=3.96$. If each of these numerical values is multiplied by $10^{11}$ then the units for constants are dynes $/ \mathrm{cm}^{2}$. Table 1 shows the variation in $(-M / N)^{\frac{1}{2}}$ as $\alpha$ assumes the values $n \pi / 18(n=1,2, \cdots, 9)$.

TABLE 1

Values of $(-M / N)^{\frac{1}{2}}$ for $\alpha=n \pi / 18$

\begin{tabular}{l|ccccccccc}
\hline$n$ & 1 & 2 & 3 & 4 & 5 & 6 & 7 & 8 & 9 \\
\hline$(-M / N)^{\frac{1}{2}}$. & .356 & .360 & .367 & .377 & .389 & .401 & .411 & .417 & .419 \\
\hline
\end{tabular}

\section{References}

[1] J. D. Eshelby, W. T. Read and W. Shockley, 'Anisotropic elasticity with applications to dislocation theory', Acta Met. 1 (1953), 251-259.

[2] A. N. Stroh, 'Dislocations and cracks in anisotropic elasticity', Phil. Mag. 3 (1958), 625-646.

[3] A. E. Green and W. Zerna, Theoretical elasticity (O.U.P. 1st edition, 1954), Chapter 9.

[4] S. G. Lekhnitskii, Theory of elasticity of an anisotropic elastic body (Holden-Day, 1963), $131-141$.

[5] J. Brilla, 'Contact problems of an elastic anisotropic half plane' (in Roumanian), Studii si Cercetari Mecan. Apl. Inst. Mecan. Appl., Acad. Rep. Pop. Romine 12, (1961), 959-987.

[6] L. A. Galin, Contact problems in the theory of elasticity (Translation by North Carolina State College Department of Mathematics, 1961), 90-111.

[7] L. M. Milne-Thomson, Plane elastic systems (Springer-Verlag, 1960), Chapter 7.

Department of Mathematics

University of Melbourne 